EPJ Web of Conferences 70, 00065 (2014)

DOI: $10.1051 /$ epjconf/ 20147000065

(C) Owned by the authors, published by EDP Sciences, 2014

\title{
The String and the Cosmic Bounce
}

\author{
V. Bozza ${ }^{1, a}$ \\ ${ }^{1}$ Department of Physics "E.R. Caianiello", University of Salerno, Italy and \\ Istituto Nazionale di Fisica Nucleare, Sezione di Napoli, Italy.
}

\begin{abstract}
String theory introduces a new fundamental scale (the string length) that is expected to regularize the singularities of classical general relativity. In a cosmological context, the Big Bang is no longer regarded as the beginning of time, but just a transition between a Pre-Big Bang collapse phase and the current expansion. We will review old and recent attempts to build consistent bouncing cosmologies inspired to string theories, discussing their solved and unsolved problems, focussing on the observables that may distinguish them from standard inflationary scenarios.
\end{abstract}

\section{Introduction}

String theory was originally conceived for an effective description of the strong interaction [1, 2]. However, it was soon realized that many of the divergences present in the Quantum Field Theory (QFT) of point-particles were absent in a theory of extended one-dimensional objects. String theory was thus regarded as a possible UV completion of QFT. The discovery that gravity was contained in the spectrum of excitations of the bosonic string made string theory the best candidate for the Theory of Everything, i.e. a unified theory of all interactions and all particles. However, the naive idea that the standard model of known particles can be extracted from first principles of string theory has been abandoned long ago. There are a huge number of possible formulations and low energy limits of string theory, which leave a great indeterminacy in the possible manifestations of string theory. Indeed, the energies at which genuine string physics come into play are close to the Planck scale and are unreachable by any conceivable accelerator machines. It is thus difficult to imagine a signature of string physics that may be visible in human experiments. At the same time, string theory is impossible to falsify by low energy experiments, given the great variety of possible realizations of physics just beyond the standard model.

Nevertheless, it was soon realized that a possible alternative way to access string physics exists [3]. In the standard view, our universe was generated in a big explosion (the big bang cosmological singularity), from which all matter, energy and spacetime itself was generated. This means that by tracing the energy density back in time we will find the "so called" Planck era, at which matter and energy are so dense that the scales of quantum and relativistic effects coincide. We do not expect General Relativity to work at such energies, since spacetime itself should behave in a quantum way. If string theory is the correct theory of everything, it might tell us how the universe came out of this

ae-mail: valboz@sa.infn.it

This is an Open Access article distributed under the terms of the Creative Commons Attribution License 2.0, which permits unrestricted use, distribution, and reproduction in any medium, provided the original work is properly cited. 
primordial Planck soup and if the big bang singularity is just an extrapolation of General Relativity in a regime where it no longer holds.

The latter idea is what brought many people to imagine scenarios in which the big bang was not the beginning of time but just a transition from a preceding universe, similar to ours or very different, depending on the scenarios [4]. The Pre-Big Bang universe ended with a collapse phase that would have inexorably lead to a big crunch singularity if it were not for the string physics. Strings are objects that behave in a strange way, since they enjoy several duality properties. For example, it is possible to relate the behavior at high energy to the behavior at low energy through the T-duality. So, it is expected that string physics might make a cosmic bounce possible, turning a big crunch into a big bang.

Is this real or just some sort of fairytale or science fiction? As physicists, we must come out with something that can be experimentally testable, which seems impossible when we speak about the Pre-Big Bang universe or the primordial soup. On the contrary, present observations are able to open a window to the very early universe through the study of cosmological perturbations [5]. The galaxies we observe today are just the outcome of the evolution of primordial fluctuations in an otherwise homogeneous universe along billions of years. Therefore, by the observations of galaxies and background radiation, we can reconstruct the primordial spectrum of cosmological perturbations, which may contain the signature of the theory of everything, being it the string theory or something else.

In the middle between Planck era and the low energy universe, there is inflation. The accelerated expansion of the universe was introduced to solve some puzzles of the standard big bang scenario (homogeneity, curvature, entropy, ...). As a bonus, inflation also provides a natural explanation of the primordial spectrum of perturbations. Is inflation compatible with string theory or not? Is it absolutely unavoidable? These questions have found different answers in string cosmology, as we will see [6,7].

In this review, we shall first recall how inflation works, how it fits the main observables related to cosmological perturbations, and which points of contact exist with String Theory. Then we shall closely consider bouncing cosmologies briefly reviewing the most promising and relevant attempts. Next we shall investigate the difficult issue of cosmological perturbations in bouncing universes. Finally, we will make a comparison between the predictions of inflation and bouncing scenarios, in search of stringy signatures hidden within the cosmological observables.

Given the extreme width of the scope, it is impossible to provide an exhaustive account of all innumerable contributions to the field in a few pages. We will just follow our path to expose our personal view, focussing on the works that are relevant to it. For more details and completeness, we refer the reader to the books and reviews cited along this introduction.

\section{The Inflation and the String}

\subsection{Theoretical need for inflation}

The standard big bang cosmology suffered from two important theoretical problems: why is the universe so homogeneous? why is the universe so flat?

The homogeneity problem stems from the observation that a particle horizon exists in FriedmanLemaitre cosmologies. In practice, a particle generated at the big bang can only travel a finite distance up to the present time. Different regions of the universe that are farther than the particle horizon have never exchanged particles all along their history. Then there is no reason why they should have similar temperature or energy density. However, the cosmic background radiation we observe today is very homogeneous even if it was generated in patches of the universe that were much more distant than the particle horizon at the time of the radiation decoupling. 
As long as the time flows, new regions become accessible to observations. In terms of Fourier components, a mode of wave number $k$ becomes accessible as $k / a H$ becomes larger than 1 . In a standard decelerated expansion, the scale factor evolves as $a \sim t^{p}$ with $p<1$ and the Hubble rate $H=\dot{a} / a \sim t^{-1}$. Thus more and more scales become accessible all along the cosmic expansion.

The flatness problem comes from the fact that the spatial curvature decays at a slower rate than the matter energy density. More precisely, the ratio of the curvature to energy density goes as $K / a^{2} H^{2}$ with $K= \pm 1$ in a positively (negatively) curved universe. So, if we do not have any evidence of spatial curvature today, this ratio must have been set to an extremely small value from the very beginning, which seems very unnatural.

Both problems can be easily solved by allowing a phase of accelerated expansion (called inflation) in the early stages of the universe, before the standard decelerated expansion sets in [8]. In fact, if $a \sim t^{p}$ with $p>1$ the ratios $k / a H$ and $K / a^{2} H^{2}$ become smaller and smaller. This means that the curvature may have started from a value comparable to the energy density and may have become tiny thanks to the inflationary phase. At the same time, regions of space that initially were in communication may have been stretched by the accelerated expansion to such extent that they could no longer communicate. So, the homogeneity paradox would be solved by the fact that different patches of the universe had the time to communicate and homogenize before inflation.

Inflation can be easily realized by invoking the existence of a scalar field that dominated the universe by its potential energy density. Depending on the shape of the potential, the number of scalar fields and other innumerable technical variations, the expansion rate can take a large number of forms.

\subsection{CMB and cosmological perturbations}

We have mentioned that the high degree of isotropy of the cosmic microwave background of radiation (CMB) was one of the reasons for the introduction of inflation. However, a perfectly homogeneous universe would have never created galaxies and stars. The fluctuations that gave rise to large scale structure in the universe are also present in the CMB in the form of anisotropies of the order of $10^{-6}$ in the temperature. These anistropies are the outcome of the evolution of a primordial spectrum of perturbation through eons of interactions between radiation and the plasma of baryons, that have created a characteristic spectrum in the multipole expansion of the anisotropies observed today [9].

By a careful study of the anisotropies in the CMB it is possible to infer the shape of the primordial spectrum of perturbations. By expressing it in terms of the Newtonian gravitational potential, it takes the form of a power law

$$
P_{\Phi}=k^{3}\left|\Phi_{k}\right|^{2}=A k^{n_{s}-1}
$$

The spectral index for scalar perturbations $n_{s}$ is constrained by CMB measurements to be $n_{s}=$ $0.963 \pm 0.012$. The precision of such measurement is an amazing result of the recent progresses in the observational cosmology, especially thanks to the WMAP spacecraft.

Note that $n_{s} \simeq 1$ corresponds to a flat (scale-invariant or Harrison-Zel'dovich) spectrum. This is excluded at the level of $3 \sigma$, at least in the simplest fits to the experimental data.

The scalar spectral index is not the only information on the primordial cosmological perturbations that can be extracted from the CMB. We can also put an upper limit on the relevance of gravitational waves. This is typically indicated by the tensor-to-scalar ratio

$$
r<0.36
$$

Whenever the tensor contribution will be observed, we might also be able to constrain the tensor spectral index $n_{T}$, which is presently unknown. 
In addition, we must also take into account the fact that cosmological perturbations may be not Gaussianly distributed. The non-Gaussianity level is encoded through other variables such as $f_{N L}$, which is also constrained by present observations:

$$
-10<f_{N L}<74
$$

More complications to the basic models of cosmological perturbations may predict running spectral index, isocurvature modes, oscillations and so on.

Summing up, we see that any model of the very early universe must provide an explanation of the theoretical puzzles of homogeneity and flatness and also provide a mechanism for the generation of the correct spectrum of primordial perturbations, without violating any of the constraints coming from the experiments.

\subsection{Inflation and perturbations}

In the inflationary scenarios, the universe expands at such a rate that every region of the size of the Hubble radius $1 / \mathrm{aH}$ finally contains no particle on average. In practice, the universe becomes empty and all that remains are quantum fluctuations of the vacuum. By a QFT formalism it is possible to calculate the spectrum of the fluctuations of the Newtonian potential in such a vacuum and it turns out to have the form of Eq. (1) with the spectral index determined by the so-called slow-roll parameters

$$
n_{s}=1-4 \epsilon-2 \delta \text {, }
$$

which depend on the specific model of inflation considered. The coincidence with the experimental value is once more fascinating. The difference $n_{s}-1$ can be used to constrain the slow-roll parameters and thus derive information on the correct inflationary model.

In the context of inflation, a similar spectrum is derived for the gravitational waves

$$
P_{h}=k^{3}|h|^{2}=A k^{n_{T}}
$$

with $n_{T}=-2 \epsilon$. A tiny level of non-Gaussianity is also predicted for the simplest models of inflation.

In definitive, besides solving theoretical puzzles, inflation also provides a natural mechanism for the generation of primordial perturbations that works in wonderful agreement with experimental constraints. So, why look for alternatives to inflation?

\subsection{Open issues in inflation}

Without diminishing its great successes, inflation still leaves some questions open, that call the need for a more accurate picture of the early universe [1].

Inflation typically needs the introduction of a new scalar field to explain the accelerated expansion. Yet, no fundamental scalar fields have been observed up to now (the Higgs field is yet to be confirmed and cannot be the inflaton anyway). So, the existence of an inflaton with a potential energy at the inflation scale is just postulated but not proved. No consistent picture or theory has been formulated in which a scalar particle can be identified with the inflaton field. One may also read this consideration the other way round: inflation works so fine that it may be taken as evidence for the existence of more scalar particles at the energy level of inflation $\left(10^{16} \mathrm{GeV}\right)$.

The second problem is that the initial singularity is not avoided by inflation. There is no way to avoid the existence of the Big Bang singularity in General Relativity. As mentioned before, this means that if we want a complete description of the very early universe, inflation is not enough and cannot 
provide any answers in this respect. The absence of a complete picture of the early universe may raise doubts on the initial conditions that have generated inflation, but inflation proves to be robust since it is a dynamical attractor. Anyway, the obscure origin of the universe calls for deeper investigations in order to put inflation on more robust ground.

The third problem concerns the mechanism of generation of primordial perturbations itself. If we trace back the scales of the perturbations we observe today, they get more and more blue-shifted because of the cosmological expansion. When we get back to inflation, they become even much smaller than the Planck length. Then how can we trust QFT on a classical spacetime at scales at which gravity itself should become quantum. The initial state of the perturbation modes is the outcome of some Transplanckian evolution that is completely unknown to us.

Finally, the potential of the inflaton must be flat enough to ensure a slow roll of the scalar field. However, whether this is fine tuning or not depends on personal points of view.

In conclusion, what we learn is that inflation is a model that works extremely well, but it is not a theory of the very early universe. The unknowns of quantum gravity, Planck energies and the initial singularity are still there and may potentially cast doubts on the beautiful successes of inflation.

\subsection{Hints from String theory}

As mentioned before [6], String theory unifies all interactions in a single consistent quantum picture. The action of String Theory depends on a single parameter: the string length $L_{S}$. This length plays the role of a cutoff of UV divergences present in conventional QFT. It also sets a natural scale separating our low energy world from truly stringy physics.

Another important ingredient comes from the fact that string theory requires supersymmetry to avoid tachyons and can be consistently formulated only in 10 (or 11) dimensions. So, every model trying to reconcile string theory with the observed universe has to give account of these extra-dimensions.

A typical solution is to assume that the extra-dimensions are compactified on a very small scale, unobservable to low energy experiments. However, there is an extremely large number of possible compactifications, which also depends on the way fluxes of p-forms are trapped in these dimensions.

In addition, the extra-degrees of freedom of the 10-dimensional fields survive after compactification in the form of scalar fields, the so-called moduli. The role of these moduli and their stabilization is also another difficult problem to face for string-inspired models.

\subsection{String Cosmology}

Since Stringy physics only plays a role at very high energies, close to the Planck scale, cosmology can help String Theory in finding experimental signatures that would be impossible to test by accelerators.

On the other way, String Theory can help cosmology in finding answers to the questions regarding the very early universe and eventually establish solid foundations for inflation.

The roads taken by string cosmologists are two: either incorporate inflation in string theory, or replace inflation by an alternative stringy cosmological phase.

As for the first option, i.e. string inflation, the field is very rich and relatively young, with the first acceptable models published after 2002. For a nice review of these models, we refer the reader to Mulryne and Ward [7]. It is interesting to note that these models make potentially testable predictions on the perturbations spectra, their indices and the level of non-Gaussianity. So, future accurate observations by PLANCK of the CMB anisotropies will probably make only a bunch of models survive [1].

In the rest of this paper we will just focus on the second option, concerning alternatives to inflation. 


\section{The String and the Bounce}

String theory has been proposed as a theory of quantum gravity, so the first thing one would like to understand is whether the big bang singularity, which is a result of classical General Relativity, persists or not in a full quantum picture. The answer is not that easy as one may expect, since nobody knows how to solve the full string theory beyond the low energy limits, which may be inadequate for such purpose.

What is typically done is to use first principles inspired to string theory to draw a heuristic picture of the universe and try to derive results that are independent of the computational details.

A first example is String Gas Cosmology [1], in which the extended nature of strings plays a crucial role. In the very early universe, all vibration modes of the strings are excited and the thermal spectrum of such string gas may generate a scale-invariant spectrum [1]. This would make this scenario compatible with observations on cosmological perturbations.

Another fascinating feature of this scenario is that it can dynamically justify the existence of three large dimensions as opposed to six very small spatial dimensions. This is obtained by observing that winding modes of strings in toroidal topologies prevent the growth of compact dimensions. However, strings can eliminate winding modes by collisions. The probability of such collisions has non-zero measure only with no more than three large spatial dimensions.

The initial singularity problem is pushed in the infinite past, as in inflationary backgrounds, by considering an emergent universe from an unstable string gas at the Hagedorn temperature.

\subsection{Bounce physics}

String theory is able to regularize UV divergences of QFT. It is natural to expect that the singularities of the classical gravitational theory such as the big bang are cured in the full quantum picture. Even if we do not know the details of the regularization, we can naturally expect that the energy scale is set by the String Mass $\left(\hbar / L_{S}\right)$. If the big bang is no longer a singularity, then we can wonder what the universe before the big bang looks like [3]. One possibility is that the big bang is actually a transition from a previous collapse phase. The collapse could not reach a big crunch singularity because of string physics, which should provide a mechanism for the transition to the post-bounce expansion.

The idea of bouncing cosmologies, as we see, contains heavy assumptions on the string physics. Everything is based on the hope that the big bang singularity is regularized. If this is not the case, then bouncing cosmologies would just be regarded as pure mathematical constructions. Nevertheless, it is worth exploring the consequences of this basic assumption and see whether a reasonable bouncing universe that can fit the experimental constraints can be built out of the bricks we already have.

The first important observation is that a contraction phase can provide a solution to the homogeneity and flatness puzzles as effectively as inflation. In fact, during an accelerated contraction, the ratios $|k / a H|$ and $|K / a H|$ shrink as during inflation.

The second encouraging point is that perturbations observed today never become TransPlanckian. In fact, tracing back perturbations in the past, they are blue-shifted until the bounce time, then they are red-shifted during the pre-bounce phase. So, the generation of perturbations from vacuum fluctuations in the asymptotic past of the bounce is much safer than in inflation.

\subsection{Challenges for bouncing cosmologies}

We have mentioned the appealing features of bouncing cosmologies, but the difficulties to be faced in order to build viable scenarios are indeed extremely hard. 
Firstly, we must be aware that a bounce in classical General Relativity requires a violation of the Null Energy Condition. Exotic matter is required, whose quantum behavior may be extremely dangerous, with the emergence of ghost degrees of freedom. This is one of the main objections to bouncing cosmologies, that often discourages most researchers. It must be said that several mechanisms are known to obtain bouncing cosmologies without the generation of ghosts, though it is not clear if they have a consistent UV completion. Indeed, if string physics is able to regularize the big bang it must also be able to provide a bounce, and this might happen in a context very different from classical General Relativity, where low energy arguments do not apply [1].

Secondly, while in an expanding universe initial anisotropies tend to become negligible, contraction makes the universe more and more anisotropic. Simple calculations in Bianchi models show that the shear grows unless the equation of state of the source driving the contraction is super-stiff, i.e. $w>1$. The danger is that the universe become shear-dominated and undergoes BKL oscillations in its final collapse phase. It is not clear whether a string phase, besides taking care of the singularity, can also tame anisotropies [1].

In third place, we must satisfy the experimental constraints on perturbations, and this limits the possible sources driving the contraction. We will examine several possibilities later on.

Finally, once we have calculated the spectrum of perturbations before the bounce, we have to infer the spectrum after the bounce. If we know nothing on the string phase, how can we propagate perturbations through the bounce? Indeed, general results are needed that make a minimal number of assumptions on the bounce.

\subsection{Models of Bouncing Cosmologies}

Historically, the first bouncing string cosmology is the Pre-Big Bang scenario by Gasperini and Veneziano [1]. This is based on the fact that String theory enjoys a T-duality, relating physics at large scales with physics at small scales. In a cosmological context, this translates into a scale-factor duality, which allows to build solutions for the Pre-Big Bang phase from Post-Big Bang solutions. The ideal self-dual solution would nicely join the Pre-Big Bang and the Post-Big Bang branches realizing a symmetric bounce (note that the universe contracts in the Einstein frame but not in the physical string frame). The spectrum of the perturbations is steeply blue $\left(n_{s}=4\right)$ in such solutions, but the axion field is endowed with a scale-invariant spectrum. Indeed, it is possible for this field to transfer its spectrum to the Newtonian potential (curvaton mechanism) and make this scenario marginally compatible with observations, though not as simple as inflation [1].

Extra-dimensions are a peculiar prediction of String theory that may play a role in a bounce. In many scenarios, the observable universe is confined to a brane. In some cases, the brane is at the boundary of a bulk warped space, in other cases it travels as a probe brane through a higher dimensional space, experiencing the curvature of such space [1].

We can also distinguish a class of High energy bounces, in which attempts have been made to directly extract a bounce from quantum gravity calculations with Loop Quantum Gravity or Wheeler-de Witt approaches [1]. In a more stringy context, higher derivative actions obtained from $\alpha^{\prime}$ corrections [2] or from non-perturbative approaches have been examined. In the latter context, interesting bounces without ghosts have been found [2].

Low energy bounces have been investigated by several mechanisms, starting from vector bounces and Weyl Integrable spacetimes [2], up to more recent non-local dilaton potential [2] and ghost condensates [2]. The last two mechanisms have also attracted interest since they seem to have no ghost at the quantum level.

Finally, we mention the Ekpyrotic/cyclic universes, in which our universe is a brane that attracts another brane until collision occurs [2]. The effective action on the visible brane just sees a scalar 
field endowed with a negative exponential potential. Then the pre-bounce phase is described by an extremely slow contraction $a \sim|t|^{p}$ with $p \ll 1$, i.e. the scalar field behaves as an extremely stiff source $w \gg 1$. In this way, there is no anisotropy problem. This scenario is interesting because of the behavior of perturbations in the pre-bounce phase, which will be thoroughly analyzed in the next section. The physics of the bounce is not clear anyway and different versions of this scenario (including the cyclic) have appeared.

\section{The Bounce and the Perturbations}

\subsection{The growing mode}

Using the well-known formalism to calculate the spectrum of quantum fluctuations in a cosmological background [2], it is possible to show that the Newtonian potential in a contracting universe dominated by a source with equation of state $p=w \rho$ is endowed with a power-law spectrum with spectral index

$$
n_{g}=1-\frac{4}{1+3 w} \text {. }
$$

It is clear that if $w \gg 1$ the spectrum of the growing mode is nearly scale-invariant, as required by the observations. The Ekpyrotic universe was then proposed as a possible challenger to inflation as a correct paradigm for the early universe [2].

However, it was soon clear that it was not at all granted that this growing mode transfers to a constant mode in the post-bounce. If this is the case, then the Ekpyrotic scenario works, if the growing mode just converts to a decaying mode, then the spectrum would be different and the scenario is no longer a valid alternative. Several toy models of bounces were analyzed in detail with conflicting results [2]. This depended on the difficulty to explicitly calculate the evolution of perturbations in a bouncing background, due to the singular behavior of many gauges at the bounce.

\subsection{A general solution for perturbations through a bounce}

Who decides whether the pre-bounce growing mode survives or not?

After examination of a large class of models [2], it has been possible to obtain a sharp conclusion on a very generic class of bounces satisfying a set of four minimal assumptions [2]:

i It makes sense to define a space-time metric even during the bounce phase.

ii The background metric is FRW.

iii The bounce is entirely determined by a single physical scale.

iv There is no inflation after the bounce or deflation before the bounce.

Assumption iii plays a crucial role, because it allows to infer the evolution of perturbations by a simple dimensional analysis of the formal solutions to the differential equations they follow.

The final result is that the fate of the growing mode depends on a very simple condition on the bounce physics. By encoding high energy corrections into an effective stress-energy tensor, the transfer of the growing mode to a post-bounce constant mode occurs if

$$
\delta p_{u} \propto \Phi
$$

where $\delta p_{u}$ is the comoving pressure perturbation of the effective source and $\Phi$ is the Newtonian potential. 
Interestingly, for perfect fluids or scalar fields, the constraint equations and the equations of state can be combined to show that $\delta p_{u} \propto \nabla^{2} \Phi$, which instead leads to the decay of the pre-bounce mode.

So, the success of the Ekpyrotic scenario in its original formulation depends on the ability of the high energy physics governing the bounce to introduce corrections to the Einstein equation directly proportional to the Newtonian potential rather than its Laplacian. With a different approach, working on junction condition with a bounce reduced to a spacelike hypersurface connecting the contraction and the expansion phase, a condition on the surface tension can be derived that can be viewed as a limit of Eq. (7) [3]. The realization of this condition may be connected to the presence of non-local microphysics at the bounce [3]. Even if Eq. (7) is not satisfied by any known cosmological source, full string theory, which introduces non-locality elements by the finite size of the strings may be able to fulfill Eq. (7). However, this is all to be proved.

\subsection{The isocurvature mode}

The transfer of the pre-bounce growing mode to the post-bounce constant mode requires heavy assumptions on the bounce physics, which is something everybody wants to avoid, as long as the bounce mechanism itself remains unclear.

A way around this problem is to use two or more scalar fields with similar negative exponential potential. Then all these fields develop similar perturbation spectra, but the existence of isocurvature modes (one for each additional field) makes the transfer of the spectrum to the constant mode granted [3]. The "New Ekpyrotic" scenario, which proposes this idea, would satisfy the requirement of a nearly scale invariant spectrum of scalar perturbations. However, it can be shown that it would produce large non-Gaussianities, already in tension with the present bounds. Furthermore, the pre-bounce phase is unstable and requires a pre-Ekpyrosis phase to tune the scalar fields on the correct trajectory [3].

\subsection{The constant mode}

What happens if we let the growing mode decay? The post-bounce spectrum is determined by a subdominant constant mode, with a spectrum

$$
n_{c}=5-\frac{4}{1+3 w} \text {. }
$$

In the Ekpyrotic limit $w \gg 1$, this would give $n_{c}=5$, which is unacceptable. However, if we choose a pre-bounce phase with $w=0$, we get $n_{c}=1$, a scale-invariant spectrum [3].

$w=0$ means a cosmological source that behaves like non-relativistic particles, the so-called dust. However, even if we start with non-relativistic particles, sooner or later they would become relativistic as the energy density of the universe grows. One may replace particles with a scalar field with exponential potential mimicking dust, but it can be shown that the tracking solution would be a repeller in a contracting universe, rather than an attractor [3].

Even worse, the anisotropy problem would not at all solved with $w=0$ and the universe would end up in chaotic BKL oscillations [3]. A possible solution to the anisotropy problem would be the existence of non-linear terms in the equation of state [3]. In fact, we may consider the linear equation of state $p=w \rho$ as the truncation of a more general expansion $p=w \rho+\beta \rho^{2}+o\left(\rho^{2}\right)$. Then, when non-linearities become important, the anisotropy becomes dramatically suppressed. This mechanism may be invoked to help dust or radiation pre-bounce phases. 


\section{The Bounce and the Inflation}

At the end of this quick overview of inflation and bouncing cosmologies, we can try to draw our conclusions by listing the positive and negative points of the different scenarios and look for possible differences in their predictions.

Both inflation and pre-bounce contraction solve the homogeneity and flatness puzzles without problems.

Transplanckian physics may be potentially dangerous for inflation but not for bouncing cosmologies.

Inflation solves the anisotropy problem as well as all bouncing cosmologies with superstiff sources $(w>1)$. Otherwise, bouncing cosmologies need additional mechanisms (e.g. non-linearity in the equation of state).

Inflation beautifully fits the experimental bounds on the scalar spectral index. Bouncing cosmologies can do it either with a $w \gg 1$ pre-bounce and an additional assumption on the bounce physics (Ekpyrotic scenario), or with a dust-dominated contraction and additional mechanisms for stabilization.

The amplitude of the gravitational wave background is small but not negligible in inflation, while it is tiny in Ekpyrotic cosmologies and comparable to scalar perturbations in dust-dominated contraction. In the Ekpyrotic case, the gravitational wave spectrum is also steeply blue.

Non-Gaussianities are generically larger in bouncing cosmologies, but can also be large in particular inflation models.

Another feature that may carry the signature of a cosmic bounce is the black hole overproduction which may have left "circles in the sky", i.e. particular traces in the cosmic background [3].

In conclusion, for bouncing cosmologies it is still hard to reproduce the great successes of inflation without inflation. Probably the two scenarios must not be viewed as alternative to each other. Inflation needs a completion at Planckian energies, where quantum gravity ruled the universe. String theory provides hints for a regularization of the singularity that naturally opens the way to bouncing scenarios. So, both the bounce and inflation may be important pieces of the history of our universe.

\section{References}

[1] M.B. Green, J.H. Schwarz, and E. Witten, Superstring Theory, Cambridge Monographs on Mathematical Physics, 1988

[2] J. Polchinski, String Theory, Cambridge Monographs on Mathematical Physics, 1998

[3] M. Gasperini, Elements of String Cosmology, Cambridge University Press, 2007

[4] M. Novello and S.E. Perez Bergliaffa, Phys. Reps. 463, (2008) 127

[5] S. Dodelson, Modern Cosmology, Academic Press, 2003

[6] C.P. Burgess and L. McAllister, Class.Quant.Grav. 28, (2011) 204002

[7] D.J. Mulryne and John Ward, Class.Quant.Grav. 28, (2011) 204010

[8] D. Langlois, Lect.Notes Phys. 800, (2010) 1

[9] E. Komatsu et al. ApJS 192, (2011) 18

[10] R.H. Brandenberger, arXiv:0902.4731

[11] www.rssd.esa.int/planck

[12] R.H. Brandenberger and C. Vafa, Nucl. Phys. B 316, (1989) 391

[13] A. Nayeri, R.H. Brandenberger, and C. Vafa, Phys. Rev. Lett. 97, (2006) 021302

[14] S. Dubovsky, T. Gregoire, A. Nicolis, and R. Rattazzi, JHEP 0603, (2006) 025

[15] Th. Damour and M. Henneaux, Phys. Rev. Lett 85, (2000) 920 
[16] G. Veneziano, Phys. Lett. B, 265, (1991) 287; M. Gasperini and G. Veneziano, Astropart. Phys. 1, (1993) 317; M. Gasperini and G. Veneziano, Phys. Rep. 373, (2003) 1

[17] K. Enqvist and M.S. Sloth, Nucl. Phys. B 626, (2002) 395; D.H. Lyth and D. Wands, Phys. Lett. B 524, (2002) 5; V. Bozza, M. Gasperini, M. Giovannini, and G. Veneziano, Phys. Lett. B 543, (2002) 14; Phys. Rev. D 67063514

[18] Y. Shtanov and V. Sahni, Phys. Lett. B 557, (2003) 1; S. Mukherji and M. Peloso, Phys. Lett. B 547, (2002) 297; C.P. Burgess, P. Martineau, F. Quevedo, and R. Rabadan, JHEP 0306, (2003) 037; Ph. Brax, D.A.Steer, JHEP 0205, (2002) 016; S. Kachru, L. McAllister, JHEP 0303, (2003) 018

[19] M. Bojowald, R. Maartens, and P. Singh, Phys.Rev. D 70, (2004) 083517; P. Peter, E.J.C. Pinho, and N. Pinto-Neto, Phys. Rev. D 75, (2007) 023516;

[20] Sh. Tsujikawa, R. Brandenberger, and F. Finelli, Phys. Rev. D 66, (2002) 083513

[21] T. Biswas, A. Mazumdar, and W. Siegel, JCAP 0603, (2006) 009

[22] M. Novello and J. Salim, Phys. Rev. D 20, (1979) 377; M. Novello, L.A.R. Oliveira, J.M. Salim, and E. Elbaz, Int. J. Mod. Phys. D 1, (1993) 641

[23] M. Gasperini, M. Giovannini, and G. Veneziano, Phys. Lett. B 569, (2003) 113; Nucl. Phys. B 694, (2004) 206

[24] P. Creminelli, M.A. Luty, A. Nicolis, and L. Senatore, JHEP 0612, (2006) 080

[25] J. Khouri, B.A. Ovrut, P.J. Steinhardt, and N. Turok, Phys. Rev. D 66, (2002) 046005; A.J. Tolley, N. Turok, and P.J. Steinhardt, Phys. Rev. D 69, (2004) 106005

[26] V.F. Mukhanov, H.A. Feldman, and R.H. Brandenberger, Phys. Reps. 215, (1992) 131

[27] P. Peter and N. Pinto-Neto, Phys. Rev. D 66, (2002) 063509; F. Finelli, JCAP 10, (2003) 011; L. Allen and D. Wands, Phys. Rev. D 70, (2004) 063515; F. Finelli, P. Peter, N. Pinto-Neto, Phys. Rev. D 77, (2008) 103508

[28] V. Bozza and G. Veneziano, Phys. Lett. B 625, (2005) 177; JCAP 09, (2005) 007

[29] V. Bozza, JCAP 02, (2006) 009

[30] R. Durrer and F. Vernizzi, Phys. Rev. D 66, (2002) 083503

[31] Ch. Chu, K. Furuta, and F. Lin, Phys. Rev. D 73, (2006) 103505

[32] E.I. Buchbinder, J. Khoury, and B.A. Ovrut, Phys. Rev. D 76, (2007) 123503

[33] K. Koyama and D. Wands, JCAP 0704, (2007) 008; K. Koyama, Sh. Mizuno, F. Vernizzi, and D. Wands, JCAP 0711, (2007) 024

[34] D. Wands, Phys. Rev. D 60, (1999) 023507; F. Finelli and R. Brandenberger, Phys. Rev. D 65, (2002) 103522; R.H. Brandenberger, arXiv:1206.4196

[35] S. Gratton, J. Khoury, P.J. Steinhardt, and N. Turok, Phys. Rev. D 69, (2004) 103505

[36] J.K. Erickson, D.H. Wesley, P.J. Steinhardt, and N.Turok, Phys. Rev. D 69, (2004) 063514

[37] V. Bozza and M. Bruni, JCAP 0910, (2009) 014

[38] V.G. Gurzadyan, R. Penrose, arXiv:1011.3706 